\title{
Prevalência de dermatoses em um ambulatório universitário no ano 2019: um estudo transversal
}

\author{
Prevalence of skin diseases in a university outpatient clinic in 2019: a cross-sectional study
}

Prevalencia de dermatosis en un ambulatorio universitario en el año 2019: un estudio transversal

Ângela Patrus ${ }^{1 *}$, Mariana Luiza Moreira ${ }^{1}$, Fernanda Vaz de Melo Bacha ${ }^{1}$, Anna Clara Colen Porto ${ }^{1}$, Gláucia dos Santos Vianna ${ }^{1}$.

\section{RESUMO}

Objetivo: Analisar a prevalência geral e de acordo com as variáveis "sexo" e "faixa etária" das dermatoses de um serviço no ano 2019 e descrever o perfil epidemiológico dos pacientes. Métodos: Trata-se de estudo transversal, que analisou prontuários de 717 pacientes atendidos no serviço de dermatologia de um ambulatório universitário de uma capital brasileira no ano de 2019. Analisou-se o perfil epidemiológico dos pacientes, considerando as variáveis: sexo, idade, profissão e naturalidade. Avaliou-se a prevalência dos diagnósticos de forma global e de acordo com faixas etárias e sexo. O estudo foi aprovado pelo Comitê de Ética e Pesquisa. Resultados: As variáveis mais prevalentes foram: sexo feminino, 20 a 59 anos, naturalidade no estado de Minas Gerais e profissões aposentado, do lar e estudante. O grupo de diagnósticos mais prevalente foi discromias, seguido por tumores epiteliais benignos, micoses superficiais, erupções eczematosas e foliculoses. Conclusão: O perfil epidemiológico encontrado, incluindo sexo, faixa-etária, profissão e naturalidade, foi condizente com literatura. Ainda, a prevalência dos principais diagnósticos diferiu conforme a variável epidemiológica dos pacientes. Este resultado condiz, de forma não ordinal, com as principais hipóteses dos demais estudos. Entretanto, a falta de trabalhos com avaliações epidemiológicas semelhantes limita a análise comparativa adequada.

Palavras-chave: Prevalência, Dermatopatias, Dermatologia, Assistência ambulatorial.

\begin{abstract}
Objective: To analyze the general prevalence and according to the variables "sex" and "age group" of dermatoses in a service in 2019 and describe the epidemiological profile of the patients. Methods: This is a cross-sectional study based on the clinical record review of 717 patients assessed by the dermatology service of a university outpatient clinic in a Brazilian capital in 2019. The epidemiological profile was described, considering: sex, age, job, and place of birth. The global prevalence of diagnosis was investigated, as well as according to age and sex. The study was approved by the Research Ethics Committee. Results: The most prevalent variables were: female gender, 20 to 59 years old, born in the state Minas Gerais and occupations retired, homemaker and student. The most prevalent group of diagnoses was dyschromias, followed by benign epithelial tumors, superficial mycoses, eczematous eruptions and follicles disorders. Conclusion: The epidemiological profile found, including sex, age, job and place of birth, was consistent with the literature. Still, the prevalence of the main diagnoses differed according to the epidemiological variable of the patients. This result is non-ordinally consistent with the main hypotheses of the other studies. However, the lack of studies with similar epidemiological assessments limits proper comparative analysis.
\end{abstract}

Key words: Prevalence, Skin diseases, Dermatology, Ambulatory care.

1 Faculdade Ciências Médicas de Minas Gerais (FCMMG), Belo Horizonte - MG.
*E-mail: angelagpatrus@gmail.com

SUBMETIDO EM: 6/2021 


\section{RESUMEN}

Objetivo: Analizar la prevalencia general y de acuerdo a las variables "sexo" y "grupo de edad" de dermatosis atendidas en un ambulatório universitario en el año 2019 y describir el perfil epidemiológico de los pacientes. Métodos: Se trata de un estudio transversal, que analizó el historial médico de 717 pacientes atendidos en el servicio de dermatología de un ambulatorio universitario de una capital brasileña en 2019. Se describió el perfil epidemiológico considerando: sexo, edad, trabajo y ciudad natal. Se investigó la prevalencia general de diagnóstico, así como de acuerdo a la edad y sexo. El estudio fue aprobado por el Comité de Ética en Investigación. Resultados: Las variables más prevalentes fueron: mujeres, 20 a 59 años, nacidas en ciudades de la provincia Minas Gerais y profesiones jubilado, ama de casa y estudiante. El grupo de diagnósticos más prevalente fue el de las discromías, seguido de los tumores epiteliales benignos, las micosis superficiales, las erupciones eccematosas y los trastornos del folículo. Conclusión: El perfil epidemiológico encontrado, incluyendo sexo, edad, trabajo y ciudad natal, fue consistente con la literatura. Aún así, la prevalencia de los principales diagnósticos difirió según la variable epidemiológica de los pacientes. Este resultado es coerente, no-ordinalmente, con las principales hipótesis de los otros estudios. Sin embargo, la falta de estudios con evaluaciones epidemiológicas similares limita un análisis comparativo adecuado.

Palabras clave: Prevalencia, Enfermedades de la piel, Dermatología, Atención ambulatoria.

\section{INTRODUÇÃO}

Dermatoses é o termo empregado para doenças que acometem pele, anexos cutâneos e mucosas. A especialidade que se dedica ao tratamento das dermatoses é a dermatologia. Especialidade essa que se ocupa do diagnóstico e da propedêutica de um espectro amplo de doenças, englobando, por exemplo, neoplasias, doenças autoimunes, infecciosas e inflamatórias. Além disso, o dermatologista trata pacientes de todas as faixas etárias, raças e etnias (MIOT HA, et al., 2018; AKHIYAT S, et al., 2020).

Percebe-se a elevada prevalência de doenças de pele nas consultas médicas, não só no Brasil, como em outros países, como França, Suécia e Estados Unidos, fato que colabora com os custos significativos que essas afecções são responsáveis no sistema de saúde (MIOT HA, et al., 2018; SOCIEDADE BRASILEIRA DE DERMATOLOGIA, 2006; CARDOSO PO, et al., 2013). Diversos estudos apontam como essas patologias interferem na qualidade de vida dos pacientes, principalmente nos doentes crônicos, sendo motivo de insegurança e limitação da execução de atividades diárias (MIOT HA, et al., 2018; CALVETTI PU, 2017; AGOSTINHO KM, et al., 2013; SINGH S, et al., 2019).

Tendo visto a relevância do tema, é contraditório que haja uma tendência a não valorização das patologias dermatológicas na literatura brasileira (CARDOSO PO, et al., 2013). As pesquisas epidemiológicas a respeito das dermatoses mais frequentes no Brasil são circunscritas à abrangência geográfica e demográfica e são escassos os trabalhos, principalmente recentes, que abordam a prevalência de dermatoses em serviços de atenção secundária à saúde (CARVALHO MTF, et al., 2008; AVANCINI J, 2018).

De acordo com um estudo conduzido pela Sociedade Brasileira de Dermatologia (SBD) (2006), a principal causa de consulta é a acne. Outros estudos brasileiros seguem o mesmo padrão desse estudo, com algumas variações, que podem ser justificadas por fatores ambientais e culturais da população avaliada (MIOT HA, et al., 2018; AVANCINI J, 2018; BRANDÃO MPAS, et al., 2020). Brandão MPAS, et al. (2020), por exemplo, descrevem que eczema foi a dermatose mais prevalente, seguido por acne. Outro dado importante é a prevalência do gênero feminino nas consultas dermatológicas, descrito tanto na literatura nacional, quanto mundial (AVANCINI J, 2018).

Diante da importância desses dados para o direcionamento de políticas públicas de saúde, verifica-se a importância de estudos como este. Brandão MPAS, et al. (2020), apontaram como uma sugestão que além do estudo da prevalência das dermatoses de forma geral, também fosse analisada a prevalência por faixa etária. Por sua vez, Agostinho KM, et al. (2013) sugeriram relacionar as dermatoses a aspectos sociodemográficos. A análise da prevalência de doenças dermatológicas de acordo com variáveis como faixa 
etária e sexo tem importância por permitir um resultado mais específico e para que futuras ações educativas possam levar em consideração em quais grupos cada doença é mais prevalente.

Dessa forma, os objetivos da pesquisa são analisar a prevalência das dermatoses nos pacientes atendidos pelo serviço de dermatologia de um ambulatório universitário em uma capital brasileira no ano de 2019, bem como descrever o perfil epidemiológico dos pacientes atendidos nesse serviço e período. Ademais, busca-se investigar a prevalência das dermatoses de acordo com as variáveis "sexo" e "faixa etária" e comparar os achados com o que está descrito na literatura.

\section{MÉTODOS}

Trata-se de estudo transversal retrospectivo. Para a coleta de dados, analisou-se prontuários informatizados de pacientes atendidos no serviço de dermatologia de um ambulatório universitário de uma capital brasileira, no ano de 2019. Cada prontuário foi analisado individualmente e foi criada uma tabela no Excel (Microsoft@), com as seguintes variáveis: número do prontuário, idade, sexo, ocupação, naturalidade, o diagnóstico ou as hipóteses diagnósticas.

Os critérios de inclusão foram: Prontuários de atendimentos no serviço de dermatologia do ambulatório universitário no ano de 2019 e os critérios de exclusão foram prontuários com dados incoerentes ou faltantes, múltiplas hipóteses diagnósticas, ausência de hipótese diagnóstica ou consultas de retorno com a mesma hipótese diagnóstica. Assim, obteve-se uma amostra de 787 atendimentos, com 715 pacientes.

Inicialmente, foi feito o perfil epidemiológico dos pacientes atendidos, considerando as variáveis: sexo, idade, profissão e naturalidade. Devido à grande variedade de naturalidades encontradas, optou-se por agrupar as cidades nos estados em que se encontram. Para Minas Gerais, dividiu-se de acordo com as regiões de planejamento, divisão adotada oficialmente pelo Governo do Estado de Minas Gerais (2010). Já as profissões foram agrupadas conforme a Classificação Brasileira de Ocupações do Instituto Brasileiro de Geografia e Estatística (IBGE) (2002).

Foi analisada a prevalência dos diagnósticos encontrados nos pacientes, sendo que uma pessoa pode ter apresentado mais de um diagnóstico. Para fins didáticos, os diagnósticos foram agrupados de acordo com a classificação de Rivitti EA (2014). A prevalência de cada diagnóstico também foi analisada de acordo com a idade e sexo. Para sexo, dividimos em feminino e masculino. Para idade, as faixas etárias: 0-9 anos; 10-19 anos; 20-59 anos; 60-69 anos; 70-79 anos e 80 anos ou mais. Nas tabelas, itens com uma prevalência pouco significativa, foram agrupados em "outros".

As variáveis categóricas foram apresentadas como frequências absolutas e relativas e as variáveis numéricas, como média \pm desvio-padrão e mediana (1ำ quartil $-3^{\circ}$ quartil). As análises foram realizadas no software $R$ versão 4.0.3.

O presente estudo foi aprovado pelo Comitê de Ética em Pesquisa da instituição, sob número CAAE: 26674519.9.0000.5134. Durante a realização do trabalho, os princípios éticos foram respeitados de acordo com a resolução 466/12 do Conselho Nacional de Saúde.

\section{RESULTADOS}

Foram analisados 787 atendimentos de 715 pacientes. Para a análise dos diagnósticos e grupos de diagnósticos foram considerados todos, de todas as consultas de cada paciente, sendo a amostra final os 715 pacientes (com um total de 1.398 diagnósticos).

A Tabela 1 apresenta a análise do perfil epidemiológico dos pacientes, apontando o sexo, idade, naturalidade e profissão dos indivíduos atendidos. A idade média foi de 48,3 anos, com 18,9 de desvio padrão, enquanto a idade mediana foi de 51 anos $(32,5-63,0)$. 
Tabela 1 - Perfil epidemiológico da amostra do estudo, levando em conta as variáveis: sexo, idade, naturalidade e profissão.

\begin{tabular}{|c|c|}
\hline Variável & Frequência (\%) \\
\hline \multicolumn{2}{|l|}{ Sexo } \\
\hline Feminino & $498(69,7)$ \\
\hline Masculino & $217(30,3)$ \\
\hline \multicolumn{2}{|l|}{ Idade } \\
\hline 0 a 9 anos & $2(0,3)$ \\
\hline 10 a 19 anos & $65(9,1)$ \\
\hline 20 a 59 anos & $412(57,6)$ \\
\hline 60 a 69 anos & $150(21,0)$ \\
\hline 70 a 79 anos & $63(8,8)$ \\
\hline A partir de 80 anos & $23(3,2)$ \\
\hline \multicolumn{2}{|l|}{ Naturalidade } \\
\hline Não informado & $16(2,2)$ \\
\hline Região central de Minas Gerais & $445(62,3)$ \\
\hline Região do Vale do Rio Doce de Minas Gerais & $86(12,0)$ \\
\hline Região do Vale do Jequitinhonha e Mucuri de Minas Gerais & $42(5,9)$ \\
\hline Região da Zona da Mata de Minas Gerais & $32(4,5)$ \\
\hline Outros Estados & $21(2,9)$ \\
\hline Região Norte de Minas Gerais & $19(2,7)$ \\
\hline Região Centro-oeste de Minas Gerais & $14(2,0)$ \\
\hline Outros Estados & $21(2,9)$ \\
\hline Região Norte de Minas Gerais & $19(2,7)$ \\
\hline Região Centro-oeste de Minas Gerais & $14(2,0)$ \\
\hline Espírito Santo & $13(1,8)$ \\
\hline Bahia & $11(1,5)$ \\
\hline São Paulo & $11(1,5)$ \\
\hline Região Sul de Minas Gerais & $4(0,6)$ \\
\hline \multicolumn{2}{|l|}{ Profissão } \\
\hline Aposentado & $150(21,0)$ \\
\hline Do lar & $100(14,0)$ \\
\hline Estudante & $75(10,5)$ \\
\hline Ocupações de prestação de serviços & $73(10,2)$ \\
\hline Ocupações das indústrias de transformação e construção civil & $41(5,7)$ \\
\hline Ocupações administrativas & $35(4,9)$ \\
\hline Ocupações técnicas, científicas, artísticas e assemelhadas & $33(4,6)$ \\
\hline Ocupações do comércio e atividades auxiliares & $30(4,2)$ \\
\hline Ocupações dos transportes e comunicações & $14(2,0)$ \\
\hline Ocupações da defesa nacional e segurança pública & $1(0,1)$ \\
\hline Desempregado & $1(0,1)$ \\
\hline Outras ocupações, ocupações mal definidas ou não declaradas & $162(22,6)$ \\
\hline
\end{tabular}

Fonte: Patrus A, et al., 2021.

A Tabela 2 elucida a prevalência dos diagnósticos das consultas atendidas, de acordo com o agrupamento realizado. Esta tabela apresenta a prevalência em todos os pacientes e de acordo com sexo. 
Tabela 2 - Análise descritiva dos grupos de diagnósticos em todos os pacientes e de acordo com sexo.

\begin{tabular}{|c|c|c|c|}
\hline Grupos diagnósticos & $\begin{array}{c}\text { Frequência (\%) } \\
\text { Total }\end{array}$ & $\begin{array}{l}\text { Frequência (\%) } \\
\text { em Mulheres }\end{array}$ & $\begin{array}{l}\text { Frequência (\%) } \\
\text { em Homens }\end{array}$ \\
\hline & $\mathrm{n}=715$ & $n=498$ & $\mathrm{n}=\mathbf{2 1 7}$ \\
\hline Sem diagnóstico & $7(1,0)$ & $6(1,2)$ & $1(0,5)$ \\
\hline Discromias & $190(26,6)$ & $157(31,5)$ & $33(15,2)$ \\
\hline Tumores Epiteliais Benignos & $171(23,9)$ & $126(25,3)$ & $45(20,7)$ \\
\hline Outros & $141(19,7)$ & $93(18,7)$ & $48(22,1)$ \\
\hline Micoses Superficiais & $135(18,9)$ & $95(19,1)$ & $40(18,4)$ \\
\hline Erupções eczematosas & $127(17,8)$ & $78(15,7)$ & $49(22,6)$ \\
\hline Foliculoses & $87(12,2)$ & $59(11,8)$ & $28(12,9)$ \\
\hline Nevos Pigmentares e Melanoma Maligno & $72(10,1)$ & $58(11,6)$ & $14(6,5)$ \\
\hline Tricoses & $71(9,9)$ & $63(12,7)$ & $8(3,7)$ \\
\hline Erupções eritematoescamosas & $71(9,9)$ & $52(10,4)$ & $19(8,8)$ \\
\hline Dermatoses por Vírus & $48(6,7)$ & $31(6,2)$ & $17(7,8)$ \\
\hline Onicoses & $46(6,4)$ & $37(7,4)$ & $9(4,1)$ \\
\hline Tumores Epiteliais Malignos & $45(6,3)$ & $29(5,8)$ & $16(7,4)$ \\
\hline $\begin{array}{l}\text { Proliferações e Tumores dos Tecidos } \\
\text { Conectivo, Adiposo, Muscular e Neural }\end{array}$ & $41(5,7)$ & $32(6,4)$ & $9(4,1)$ \\
\hline Erupções papulopruriginosas & $40(5,6)$ & $28(5,6)$ & $12(5,5)$ \\
\hline $\begin{array}{l}\text { Reações a Agentes Mecânicos, Calor e } \\
\text { Frio }\end{array}$ & $28(3,9)$ & $19(3,8)$ & $9(4,1)$ \\
\hline Afecções atrófico-escleróticas & $23(3,2)$ & $18(3,6)$ & $5(2,3)$ \\
\hline Cistos & $22(3,1)$ & $13(2,6)$ & $9(4,1)$ \\
\hline $\begin{array}{l}\text { Piodermites e outras Dermatoses por } \\
\text { Bactérias }\end{array}$ & $20(2,8)$ & $13(2,6)$ & $7(3,2)$ \\
\hline Afecções do tecido conectivo & $13(1,8)$ & $11(2,2)$ & $2(0,9)$ \\
\hline
\end{tabular}

Fonte: Patrus A, et al., 2021.

Com relação à prevalência dos grupos diagnósticos de acordo com as faixas etárias, tem-se 2 pacientes com 0 a 9 anos, sendo que 1 possuía diagnóstico do grupo "Erupções eczematosas" e 1 do grupo "Erupções eritematoescamosas".

Na Tabela 3 está exposta a análise dos diagnósticos mais prevalentes de acordo com a faixa etária, com uma coluna para 10 a 19 anos e outra para 20 a 59 anos. 
Tabela 3 - Análise descritiva dos grupos de diagnósticos dos pacientes com 10 a 19 anos e com 20-59 anos.

\begin{tabular}{lcc}
\hline \multicolumn{1}{c}{ Grupos diagnósticos } & $\begin{array}{c}\text { Frequência (\%) } \\
\text { em 10 a 19 anos }\end{array}$ & $\begin{array}{c}\text { Frequência (\%) } \\
\text { em 20 a 59 anos }\end{array}$ \\
\cline { 2 - 3 } & $\mathbf{n = 6 5}$ & $\mathbf{n = 4 1 2}$ \\
\hline Sem diagnóstico & $3(4,6)$ & $2(0,5)$ \\
\hline Discromias & $10(15,4)$ & $124(30,1)$ \\
\hline Outros & $9(13,8)$ & $80(19,4)$ \\
\hline Erupções eczematosas & $13(20,0)$ & $73(17,7)$ \\
\hline Micoses Superficiais & $8(12,3)$ & $73(17,7)$ \\
\hline Tumores Epiteliais Benignos & $0(0,0)$ & $72(17,5)$ \\
\hline Foliculoses & $31(47,7)$ & $54(13,1)$ \\
\hline Nevos Pigmentares e Melanoma Maligno & $2(3,1)$ & $51(12,4)$ \\
\hline Tricoses & $4(6,2)$ & $51(12,4)$ \\
\hline Erupções eritematoescamosas & $1(1,5)$ & $49(11,9)$ \\
\hline Onicoses & $2(3,1)$ & $31(7,5)$ \\
\hline Erupções papulopruriginosas & $1(1,5)$ & $27(6,6)$ \\
\hline Dermatoses por Vírus & $4(6,2)$ & $25(6,1)$ \\
\hline Proliferações e Tumores dos Tecidos Conectivo, & Adiposo & $20(4,9)$ \\
Muscular e Neural & $0(0,0)$ & $20(16(3,9)$ \\
\hline Piodermites e outras Dermatoses por Bactérias & $1(1,5)$ & $15(3,6)$ \\
\hline Tumores Epiteliais Malignos & $0(0,0)$ & $14(3,4)$ \\
\hline Afecções atrófico-escleróticas & $5(7,7)$ & $12(2,9)$ \\
\hline Cistos & $1(1,5)$ & $12(2,9)$ \\
\hline Reações a Agentes Mecânicos, Calor e Frio & $2(3,1)$ & $11(2,7)$ \\
\hline Hidroses & $3(4,6)$ & \\
\hline
\end{tabular}

Fonte: Patrus A, et al., 2021.

Já a Tabela 4 evidencia os diagnósticos mais prevalentes em pacientes com 60 a 69 anos, 70 a 79 anos e maiores de 80 anos.

Tabela 4 - Análise descritiva dos grupos de diagnósticos dos pacientes com 60 a 69 anos, 70 a 79 anos e 80 anos ou mais.

\begin{tabular}{lccc}
\hline \multicolumn{1}{c}{ Grupos diagnósticos } & $\begin{array}{c}\text { Frequência (\%) em } \\
\mathbf{6 0 - 6 9} \text { anos }\end{array}$ & $\begin{array}{c}\text { Frequência (\%) } \\
\text { em 70 a 79 anos }\end{array}$ & $\begin{array}{c}\text { Frequência (\%) em } \\
\mathbf{8 0} \text { anos ou mais }\end{array}$ \\
\cline { 2 - 4 } & $\mathbf{n = 1 5 0}$ & $\mathbf{n = 6 3}$ & $\mathbf{n = 2 3}$ \\
\hline Sem diagnóstico & $\mathbf{2 ( 1 , 3 )}$ & $0(0,0)$ & $0(0,0)$ \\
\hline Tumores Epiteliais Benignos & $56(37,3)$ & $31(49,2)$ & $12(52,2)$ \\
\hline Micoses Superficiais & $40(26,7)$ & $10(15,9)$ & $4(17,4)$ \\
\hline Discromias & $34(22,7)$ & $16(25,4)$ & $6(26,1)$ \\
\hline Erupções eczematosas & $25(16,7)$ & $10(15,9)$ & $5(21,7)$ \\
\hline Tumores Epiteliais Malignos & $15(10,0)$ & $11(17,5)$ & $4(17,4)$ \\
\hline Dermatoses por Vírus & $14(9,3)$ & $3(4,8)$ & $2(8,7)$ \\
\hline Erupções eritematoescamosas & $13(8,7)$ & $10(15,9)$ & $0(0,0)$ \\
\hline Tricoses & $13(8,7)$ & $0(0,0)$ & $0(0,0)$ \\
\hline $\begin{array}{l}\text { Proliferações e Tumores dos Tecidos } \\
\text { Conectivo, Adiposo, Muscular }\end{array}$ & $12(8,0)$ & $7(11,1)$ & $2(8,7)$ \\
Neural & & & $1(4,3)$ \\
\hline $\begin{array}{l}\text { Nevos Pigmentares } \\
\text { Maligno }\end{array}$ & $11(7,3)$ & $7(11,1)$ & $1(4,3)$ \\
\hline Onicoses & $9(6,0)$ & $3(4,8)$ & $12(52,2)$ \\
\hline Outros & $57(38,0)$ & $28(44,4)$ & \\
\hline Fonter Pantrus A, & &
\end{tabular}

Fonte: Patrus A, et al., 2021. 


\section{DISCUSSÃO}

Quanto ao perfil epidemiológico dos pacientes analisados, a maior prevalência de pacientes do sexo feminino $(69,7 \%)$, concorda com os resultados encontrados em outros cinco trabalhos conduzidos no Brasil (SBD, 2006; AGOSTINHO KM, et al., 2013; BRANDÃO MPAS, et al., 2020; ALVES GB, et al., 2007). Esse dado discorda, entretanto, de uma pesquisa internacional realizada por Nahass G, et al. (1995) que indicou um padrão equivalente de atendimento entre os sexos, com discreta maioria na população masculina (55\%). O desfecho condiz com a demanda constante de cuidados com o corpo feminino e maior atenção das mulheres em relação ao zelo com a saúde, especificamente no Brasil (AGOSTINHO KM, et al., 2013).

O predomínio de atendimento em pacientes de 20 a 59 anos (57,6\%) corrobora com a estatística indicada por alguns estudos (SBD, 2006; BRANDÃO MPAS, et al., 2020; ALVES GB, et al., 2007). Esse resultado conflui com a maior mobilidade dessa faixa etária e preocupações com a senilidade futura. Em uma pesquisa realizada em Mato Grosso que analisou 106 prontuários atendidos em uma unidade básica de saúde, foi identificada a maior prevalência de pacientes de $0-9$ anos $(55,1 \%)$, o que pode indicar um cuidado concomitante das crianças e seus pais na atenção básica (AGOSTINHO KM, et al., 2013).

Pacientes aposentados $(21,1 \%)$, seguidos de do lar $(14,1 \%)$ e estudantes $(10,6 \%)$ foram as profissões mais prevalentes. Foi identificado um padrão laboral semelhante, com o destaque para estudantes em 19\% dos atendimentos em Brandão MPS, et al. (2020). Esse resultado pode sugerir uma maior disponibilidade de atenção à saúde em indivíduos que teoricamente apresentam menos obrigações e maior disponibilidade de tempo.

Com $26,6 \%$, a dermatose mais prevalente dentre a amostra de pacientes foi o grupo de discromias, que incluiu os principais diagnósticos: melanose solar, melasma, leucodermia solar, discromia pós-inflamatória, vitiligo, pitiríase alba, efélides, lentigo solar, dermatite ocre, hipomelanose, máculas raciais e cicatriz hipercrômica ou acrômica.

A pesquisa conduzida pela SBD (2006) avaliou as principais causas de consultas a dermatologistas a partir do cadastro de profissionais especialistas e de ambulatórios universitários com atendimento pelo Sistema Único de Saúde (SUS) de todo o país, com uma amostra de 57.343 pacientes. Nela, os transtornos da pigmentação ocupam a terceira posição de prevalência dentre as consultas, com $8,7 \%$. Já em um trabalho realizado no ambulatório de dermatologia da Universidade do Sul de Santa Catarina (UNISUL), em que foram analisados 1005 prontuários, de 537 pacientes, as discromias correspondem a 5,49\%, como sétimo diagnóstico mais prevalente (ALVES GB, et al., 2007).

Em Brandão MPS, et al. (2020), um estudo baseado nos dados de prontuários pertencentes à um ambulatório universitário, com uma amostra de 659 pacientes, as discromias não estavam presentes entre os sete diagnósticos mais comuns, não sendo informada a sua prevalência. Por sua vez, em um estudo de análise do perfil nosológico de consultas dermatológicas no território brasileiro de 2018, as dermatoses apresentavam a seguinte prevalência: melasma $(3,7 \%)$, vitiligo $(1,6 \%)$, lentigo solar $(0,9 \%)$, hipercromia pós inflamatória $(0,7 \%)$ e pitiríase alba $(0.2 \%)$, totalizando uma prevalência para o grupo de discromias de $7,1 \%$ (MIOT HA, et al., 2018).

O grupo de tumores epiteliais benignos ocupa o segundo lugar dentre os diagnósticos com $23,9 \%$, que inclui ceratose seborreica, ceratose actínica, dermatose papulosa nigra, hiperplasia de glândulas sebáceas, espiradenoma, tumor benigno de anexo. O estudo conduzido pela SBD (2006), que analisou os diagnósticos com base no código da Classificação Internacional de Doenças (CID-10), evidenciou que a ceratose actínica foi a terceira dermatose mais prevalente, dentre as vinte cinco principais dermatoses, com prevalência de $5,1 \%$. Da mesma forma, em outro trabalho semelhante, a ceratose actínica ocupou o quarto lugar, com 8,9\%, enquanto a ceratose seborreica ocupou o décimo terceiro lugar, com 3,1\%, totalizando uma prevalência de $12 \%$ de tumores epiteliais benignos (MIOT HA, et al., 2018). Já em Brandão MPS, et al. (2020), a ceratose actínica foi registrada em terceiro lugar, com prevalência de 8,9\%, e outros tumores benignos em quinto lugar, com 8\%. 
Um fato que pode contribuir para a não concordância encontrada entre os resultados deste trabalho e demais estudos é que a média de diagnósticos por paciente encontrada neste foi de 2,17, valor acima da média de outros trabalhos: 1,23, 1,21, 1,49, 1,47 (SBD, 2006; ALVES GB, et al., 2007; BRANDÃO MPAS, et al., 2020; MIOT HA, et al., 2018). Ademais, alguns dos estudos comparados utilizaram o código da CID-10 como critério para diagnóstico. Já este estudo, para uma análise mais didática, agrupou os diagnósticos em grupo de diagnósticos, que incluem mais de um código da CID-10, o que eleva a prevalência encontrada por grupo.

As micoses superficiais são a terceira dermatose mais prevalente, com 18,9\%. Os diagnósticos mais relevantes foram onicomicose, pitiríase versicolor e dermatofitoses. Em Alves GB, et al. (2007), estudo que analisou uma população atendida no serviço de dermatologia de um ambulatório, as micoses superficiais também foram o terceiro agrupamento mais prevalente, com $8,6 \%$ dos diagnósticos. Outra pesquisa que também analisou um serviço de dermatologia de um ambulatório encontrou que as micoses eram responsáveis por 7,4\% dos atendimentos (BRANDÃO MPAS, et al., 2020).

Já no estudo da SBD (2006) esse diagnóstico ficou em segundo lugar, com 8,7\% de prevalência. Enquanto na pesquisa dessa mesma sociedade de 2018, foi encontrada uma prevalência de 4,5\% (MIOT HA, et al., 2018). Verifica-se, pois, que o encontrado foi superior ao descrito na literatura. Um fator que pode contribuir para essa diferença é que, possivelmente, há mais encaminhamentos de pacientes com micoses superficiais para o ambulatório em questão, ao invés de serem resolvidos na atenção primária.

No sexo feminino, a dermatoses mais encontradas foram discromias $(31,5 \%)$, tumores epiteliais benignos $(25,3 \%)$, micoses superficiais (19,1\%), erupções eczematosas $(15,7 \%)$, tricoses $(12,7 \%)$. Entre as discromias, lesões relacionadas à fotoexposição como melanose solar $(31,6 \%)$, melasma $(27,4 \%)$, leucodermia solar $(14,7 \%)$ e hipercromia pós-inflamatórias $(14,4 \%)$ foram as mais prevalentes. Esse dado indica uma maior tendência a dermatoses vinculadas a fotoexposição no sexo feminino, o que é corroborado pelo achado de outros estudos (LOPES L, et al., 2010; FILONI A, et al., 2019). Ainda, o melasma apresenta etiologia parcialmente hormonal, relacionada a gestação e uso de anticoncepcionais orais, o que o torna mais específico e prevalente entre mulheres, contribuindo para a predominância de discromias no sexo feminino (HANDEL A, et al., 2014, MORAES AS, et al., 2021).

Já no sexo masculino, as discromias estão em quarta colocação, com $15,2 \%$. Os tumores epiteliais benignos se mantém em segundo lugar, com $20,7 \%$, seguidos pelas micoses superficiais, com $18,4 \%$. As erupções eczematosas $(22,6 \%)$, por sua vez, foram as dermatoses mais prevalentes. Foliculoses foram a quinta dermatose mais prevalente, com 12,9\%. Entre os eczemas, as dermatites de contato corresponderam a 40,9\% dos diagnósticos. Entretanto, esse resultado contradiz o que é relatado por uma metanálise, que indica maior frequência de eczemas de contato no sexo feminino do que no sexo masculino (ALINAGHI F, et al., 2019). Uma revisão sistemática publicada em 2020 atesta que há resultados controversos na literatura em relação à estatística de acne nos sexos, não sendo possível estabelecer em qual sexo essa dermatose é mais prevalente (HENG AHS, et al., 2020).

Em Lopes L, et al. (2010), estudo realizado em ambiente ambulatorial, as dermatoses mais prevalentes em ambos os sexos foram doenças infecciosas (21,65\%), eczemas (14,65\%), afecções eritematodescamativas $(9,40 \%)$, tumores benignos e cistos $(8,64 \%)$ e discromias $(7,37 \%)$. Esses dados corroboram parcialmente com os achados encontrados.

Em relação aos diagnósticos mais prevalentes por faixa etária, não foram encontrados muitos artigos que fizessem essa associação. Neste estudo, na faixa etária de 0 a 9 anos, tem-se apenas 2 pacientes, o que impossibilita uma análise quanto à prevalência de dermatoses nesse grupo. Já de 10 a 19 anos, o grupo de diagnósticos mais prevalente entre os 65 pacientes foi foliculoses, com um percentual de 47,7\%. Nesse grupo, a acne foi a patologia mais prevalente (OGE LK, et al., 2019; HENG AHS, et al., 2020). Após foliculoses, temse $\circ$ grupo erupções eczematosas, que engloba as dermatites e xerose, dentre outras patologias e o grupo discromias, que agrupa melanose solar, melasma, leucodermia solar, hipercromia pós-inflamatória e vitiligo. O estudo da SBD (2006) apresentou acne como diagnóstico mais prevalente na idade até 15 anos, com $13,9 \%$, seguido de dermatite atópica, com 13,7\%, patologia englobada no grupo erupções eczematosas. 
Na faixa etária de 20 a 59 anos, 412 indivíduos, o grupo de diagnósticos mais prevalente foi discromias, seguido por erupções eczematosas, micoses superficiais, tumores epiteliais benignos e foliculoses. $\mathrm{Na}$ pesquisa conduzida pela SBD em 2018, a faixa etária de 25 a 59 anos predominou o fotoenvelhecimento (10,5\%), que pode provocar lesões dentro do grupo discromias, seguido por melasma $(6,6 \%)$, patologia incluída no grupo discromias (MIOT HA, et al., 2018). Já em outro estudo, o principal diagnóstico em pacientes de 15 a 39 anos foi acne $(26,6 \%)$, seguido por transtornos de pigmentação $(9,4 \%)$, enquanto em pacientes de 40 a 64 anos, transtornos de pigmentação ocupou o primeiro lugar, com 11,6\% (SBD, 2006). Apesar do percentual encontrado neste estudo ter sido mais significativo (30\%), percebe-se a prevalência das discromias nesse grupo.

Nas faixas etárias 60-69 anos; 70-79 anos e 80 anos ou mais, o grupo mais prevalente foi tumores epiteliais benignos. As micoses superficiais e as discromias também tiveram representatividade nessas faixas. Já os diagnósticos do grupo tumores epiteliais malignos corresponderam a 10\%, 17,5\% e 17,4\% em cada faixa etária. É importante comentar que os tumores epiteliais benignos têm forte associação com exposição solar, principalmente crônica. Assim, é compreensível e esperado que sua prevalência seja maior em idades mais avançadas. Entretanto, é essencial atentar-se que alguns, como a ceratose actínica, constituem-se lesões pré-malignas, o que ressalta a importância do seu diagnóstico precoce e prevenção (CASARI A, et al., 2018).

No artigo conduzido pela SBD de 2018, os diagnósticos mais prevalentes em maiores de 60 anos foram câncer de pele não melanoma (19,3\%), patologia englobada em tumores epiteliais malignos, ceratose actínica $(12,9 \%)$; tumores epiteliais benignos; fotoenvelhecimento $(8,7 \%)$, que pode provocar lesões dentro do grupo discromias; ceratose seborreica $(6,2 \%)$, do grupo tumores epiteliais benignos; psoríase $(4,8 \%)$, do grupo erupções eritematoescamosas e micoses superficiais (4,8\%), do grupo de mesmo nome (MIOT HA, et al., 2018). Já uma pesquisa que analisou 80 idosos em São Paulo, apresentou-se como principal diagnóstico melanose solar (90\%), patologia do grupo discromias, e xerose de pele $(58,75 \%)$, patologia do grupo erupções eczematosas (MARIANI AC, et al., 2019).

A escassez de trabalhos recentes acerca da prevalência de dermatoses nos serviços de atenção secundária à saúde foi um fator limitante do presente estudo. Foi necessária a utilização de referências menos atuais, porém com metodologia semelhante, para que a análise comparativa pudesse ser realizada adequadamente. Dessa forma, salientamos a importância da produção de trabalhos que discorram sobre a temática, considerando a relevância do tema.

\section{CONCLUSÃO}

A análise do perfil epidemiológico dos pacientes atendidos pelo serviço de dermatologia de um ambulatório universitário no ano de 2019, incluindo sexo, faixa-etária, profissão e naturalidade, foi condizente, de forma não ordinal, com as principais hipóteses encontradas na literatura. A prevalência dos principais diagnósticos diferiu conforme a variável epidemiológica dos pacientes. Vale destacar que a principal limitação do estudo foi a escassez de dados epidemiológicos comparativos atualizados, de modo que a consulta a estudos menos recentes com metodologia semelhante foi necessária para análise comparativa adequada. Nesse sentido, ressalta-se a importante contribuição deste trabalho ao acesso a dados epidemiológicos atuais.

\section{AGRADECIMENTOS E FINANCIAMENTO}

Agradecemos à Raquel Cafaro Marinho pela ajuda com a estatística e elaboração de tabelas. À Faculdade Ciências Médicas de Minas Gerais, por dispor de apoio ao longo da iniciação científica e pelo suporte na revisão da escrita do artigo científico.

\section{REFERÊNCIAS}

1. AGOSTINHO KM, et al. Doenças dermatológicas frequentes em unidade básica de saúde. Cogitare Enferm, 2013; 18(4): 715-21. 
2. AKHIYAT S, et al. Why dermatology is the second least diverse specialty in medicine: How did we get here? Clin Dermatol, 2020; 38(3): 310-315.

3. ALINAGHI F, et al. Prevalence of contact allergy in the general population: A systematic review and meta-analysis. Contact Dermatitis, 2019; 80(2): 77-85.

4. ALVES GB, et al. Prevalência das dermatoses no ambulatório de dermatologia da UNISUL. Arq Catarin Med, 2007; 36(1):65-68.

5. AVANCINI J, et al. Prevalence of dermatoses in patients referred for evaluation in an outpatient clinic of specialties. An. Bras. Dermatol, 2018; 93(4): 513-516.

6. BRANDÃO MPAS, et al. Prevalência de dermatoses atendidas em um ambulatório universitário. Revista Interdisciplinar Ciências Médicas, 2020; 4(1): 31-36.

7. CALVETTI PU, et al. Aspectos biopsicossociais e qualidade de vida de pessoas com dermatoses crónicas. Psic., Saúde \& Doenças, 2017; 18(2): 297-307.

8. CARDOSO PO, et al. Perfil epidemiológico das doenças dermatológicas em Centro de Saúde de Atenção Primária. Rev Med Minas Gerais, 2013; 23(2): 169-172.

9. CARVALHO MTF, et al. Estudo nosológico das dermatoses diagnosticadas no hospital da Universidade Federal de Juiz de Fora. HU Revista, 2008; 34(4): 267-272.

10. CASARI A, et al. Actinic Keratosis and Non-Invasive Diagnostic Techniques: An Update Biomedicines, $2018 ; 6(1): 8$.

11. FILONI A, et al. Melasma: How hormones can modulate skin pigmentation. J Cosmet Dermatol, 2019; 18(2): 458-463.

12. GOVERNO DO ESTADO DE MINAS GERAIS. Regiões de Planejamento. Minas Gerais, 2010. Disponível em: https://www.mg.gov.br/conteudo/conheca-minas/geografia/regioes-de-planejamento. Acesso em: 20 nov. 2018.

13. HANDEL A, et al. Melasma: a clinical and epidemiological review. An Bras Dermatol, 2014; 89(5):771-82.

14. HENG AHS, et al. Systematic review of the epidemiology of acne vulgaris. Sci Rep, 2020; 10(1):5754.

15. INSTITUTO BRASILEIRO DE GEOGRÁFIA E ESTATÍSTICA (IBGE). Classificação Brasileira de Ocupações (CBO). Brasil, 2002. Disponível em: https://concla.ibge.gov.br/estrutura/ocupacao-estrutura. Acesso em: 20 nov. 2018

16. LOPES $L$, et al. Avaliação da frequência de dermatoses no serviço ambulatorial de dermatologia. An Bras Dermatol, 2010; 85(2): 264-66.

17. MARIANI AC, et al. Cuidados cutâneos e dermatoses prevalentes em idosos atendidos em uma clínica privada e sua relação com comorbidades. Rev Bras Geriatr Gerontol, 2019; 22(6): 1-9.

18. MIOT HA, et al. Profile of dermatological consultations in Brazil. An Bras Dermatol, 2018; 93(6): 916-28.

19. MORAES AS, et al. Melasma na gestação e suas medidas terapêuticas. Revista Eletrônica Acervo Saúde, 2021; 13(3): e6610.

20. NAHASS G, et al. Prevalence of cutaneous findings in hospitalized medical patients. J Am Acad Dermatol, 1995; 33(2): 207-11.

21. OGE LK, et al. Acne Vulgaris: Diagnosis and Treatment. Am Fam Physician, 2019; 100(8): 475-484.

22. RIVITTI EA. Manual de Dermatologia Clínica de Sampaio e Rivitti. 1aed. São Paulo: Artes Médicas, 2014; 745p.

23. SINGH S, et al. Quality of life among dermatology patients: a systematic review of investigations using qualitative methods. G Ital Dermatol Venereol, 2019; 154(1): 72-78.

24. SOCIEDADE BRASILEIRA DE DERMATOLOGIA (SBD). Perfil nosológico das consultas dermatológicas no Brasil. An Bras Dermatol, 2006; 81(6): 549-58. 